\title{
CHONDROCYTE EXPANSION IS ASSOCIATED WITH LOSS OF PRIMARY CILIA AND DISRUPTED HEDGEHOG SIGNALLING
}

\author{
C.L. Thompson ${ }^{1}$, J.C.T. Plant ${ }^{1}$, A.K.T. Wann², C.L. Bishop ${ }^{3}$, P. Novak ${ }^{1}$, H.M. Mitchison ${ }^{4}$, P.L. Beales ${ }^{4,6}$, J.P. \\ Chapple $^{5}$ and M.M. Knight ${ }^{1}$
}

${ }^{1}$ Institute of Bioengineering, School of Engineering and Materials Science, Queen Mary University of London, London E1 4NS, UK

${ }^{2}$ Kennedy Institute of Rheumatology, University of Oxford, Oxford OX3 7FY, UK

${ }^{3}$ Centre for Cell Biology and Cutaneous Research, Blizard Institute, Barts and The London School of

Medicine and Dentistry, Queen Mary University of London, London E1 EAT, UK

${ }^{4}$ Genetics and Genomic Medicine, UCL Great Ormond Street Institute of Child Health, London WC1N 1EH, UK

${ }^{5}$ Centre for Endocrinology, William Harvey Research Institute, School of Medicine and Dentistry, Queen Mary University of London, London EC1M 6BQ, UK

${ }^{6}$ NIHR Great Ormond Street Hospital Biomedical Research Centre, 30 Guilford Street, London WC1N 1EH, UK

\begin{abstract}
Tissue engineering-based therapies targeting cartilage diseases, such as osteoarthritis, require in vitro expansion of articular chondrocytes. A major obstacle for these therapies is the dedifferentiation and loss of phenotype accompanying chondrocyte expansion. Recent studies suggest that manipulation of hedgehog signalling may be used to promote chondrocyte re-differentiation. Hedgehog signalling requires the primary cilium, a microtubule-based signalling compartment, the integrity of which is linked to the cytoskeleton. We tested the hypothesis that chondrocyte dedifferentiation caused alterations in cilia expression that influence hedgehog responsiveness.

In vitro chondrocyte expansion to passage 5 (P5) was associated with increased actin stress fibre formation, dedifferentiation and progressive loss of primary cilia, compared to primary (P0) cells. P5 chondrocytes exhibited $\sim 50 \%$ fewer cilia with a reduced median length. Cilia loss was associated with disruption of ligandinduced hedgehog signalling, such that P5 chondrocytes did not significantly regulate the expression of hedgehog target genes (GLI1 and PTCH1). This phenomenon could be recapitulated by applying $24 \mathrm{~h}$ cyclic tensile strain, which reduced cilia prevalence and length in $\mathrm{P} 0$ cells. $\mathrm{LiCl}$ treatment rescued cilia loss in P5 cells, partially restoring hedgehog signalling, so that GLI1 expression was significantly increased by Indian hedgehog.

This study demonstrated that monolayer expansion disrupted primary cilia structure and hedgehog signalling in association with chondrocyte dedifferentiation. This excluded the possibility to use hedgehog ligands to stimulate re-differentiation without first restoring cilia expression. Furthermore, primary cilia loss during chondrocyte expansion would likely impact other cilia pathways important for cartilage health and tissue engineering, including transforming growth factor (TGF), Wnt and mechanosignalling.
\end{abstract}

Keywords: Chondrocyte, dedifferentiation, re-differentiation, primary cilia, cilium, hedgehog, lithium chloride.

*Address for correspondence: Dr Clare L. Thompson, Institute of Bioengineering and School of Engineering and Materials Science, Queen Mary, University of London, Mile End Road, London E1 4NS, UK.

Tel: +44 (0)2078823390Ｅmail: clare.1.thompson@qmul.ac.uk

\section{Introduction}

Articular cartilage has a limited capacity for repair, mostly due to its complex anisotropic structure and avascularity. Consequently, trauma to the articular surface often results in development of osteoarthritis $(\mathrm{OA})$, a degenerative cartilage disease. Current surgical treatments utilise tissue engineering approaches to repair cartilage lesions and prevent further cartilage degradation. Autologous chondrocyte implantation (ACI), perhaps considered the most successful treatment for focal lesions, requires the removal and expansion of patient chondrocytes (Rai et al., 2017). In a second surgery, these autologous cells are re-implanted, in combination with a membrane or matrix scaffold, to repair a cartilage defect. However, 
upon monolayer expansion, articular chondrocytes undergo dedifferentiation, such that expression of the chondrogenic matrix molecules, aggrecan (ACAN) and collagen type II (COL2) are reduced (Holtzer et al., 1960; Benya et al., 1978). Consequently, defects repaired in this way often heal as fibrocartilage, which is mechanically insufficient and cannot support long term recovery, merely delaying the need for total knee arthroplasty. Thus, strategies targeting either the prevention of dedifferentiation or the redifferentiation of expanded cells aim to improve the quality of the tissue generated by these approaches.

The phenotypic changes associated with dedifferentiation in monolayer culture are widely attributed to alterations to the cytoskeleton, which occur with expansion, specifically an increase in actin stress fibre formation (Benya, 1988; Parreno et al., 2017). Indeed, depolymerisation of the actin cytoskeleton in expanded cells results in alterations in cell shape and increased expression of cartilage matrix molecules (Benya, 1988; Parreno et al., 2017). The disruption of the actin filaments will influence a plethora of systems and cellular processes, such as proliferation, migration and mechanotransduction. Moreover, several studies have shown that changes to the actin filament network influence primary cilia expression and length (Kim et al., 2010; Sharma et al., 2011).

The primary cilium is a microtubule-based signalling compartment present in most cell types, including chondrocytes (Haycraft and Serra 2008). The cilium comprises a basal body, which arises from the cell's "mother" centriole and nucleates the formation of a ' $9+0$ ' microtubular scaffold, which is sheathed in a specialised membrane to form the ciliary axoneme. Protein entry into this compartment is tightly regulated at the base of the cilium, thus, a unique signalling compartment is formed whereby proteins can be shuttled into and out of the axoneme by a process called intraflagellar transport (IFT, for review see Veland et al., 2009). Cilia integrity is essential for the function of a rapidly expanding list of pathways that include growth factor signalling (Schneider et al., 2005; Zhu et al., 2009; Christensen et al., 2012), mechanotransduction (Praetorius and Spring 2001; Wann et al., 2012) and inflammation (Wann and Knight, 2012; Wann et al., 2014). Earlier reports also show the effects of extended culture and cellular senescence on primary cilia with conflicting results. Breslin et al. (2014) report an increased frequency and length of cilia in senescent fibroblasts, while Bishop et al. (2010) show that senescence is associated with cilia loss in epithelial cells. However, both studies show the downregulation of hedgehog signalling components during chondrocyte expansion. Therefore, in this study, we examined the expression of cilia in the context of chondrocyte expansion.

Growth factors, such as insulin growth factor-I (IGF-I), bone morphogenic protein-2 (BMP-2), transforming growth factor- $\beta$ (TGF- $\beta$ ), platelet derived growth factor (PDGF) and hedgehog (Hh) are employed to improve the quality of tissue engineered cartilage (Zimber et al., 1995; Lohmann et al., 2000; Kellner et al., 2002; Ko et al., 2012). These factors influence cartilage growth and metabolism through their effects on chondrocyte proliferation and differentiation and, intriguingly, are all linked to primary cilia (Huangfu et al., 2003; Schneider et al., 2005; Zhu et al., 2009; Christensen et al., 2012).

Hedgehog signalling is perhaps the most wellcharacterised of the cilia-mediated signalling pathways and it has been shown to both increase cartilage matrix production in tissue engineered constructs (Kellner et al., 2002) and promote chondrocyte re-differentiation (Lin et al., 2014). In the absence of hedgehog ligands, full-length Gli transcription factors (Gli2 and Gli3) traffic through the ciliary compartment and undergo protein kinase A (PKA)-dependent phosphorylation, resulting in proteolysis and production of the transcriptional repressor Gli3R (Sasaki et al., 1999; Huangfu et al., 2003). In the presence of hedgehog ligands, the transmembrane protein smoothened (smo) traffics into the cilium (Corbit et al., 2005). PKA activity is inhibited by the removal of the G-protein coupled receptor (GPCR) Gpr161 from the cilium and consequently full-length Gli activators are formed (Mukhopadhyay et al., 2013; Nager et al., 2017). Activated Gli proteins enter the nucleus and promote the transcription of hedgehog target genes, such as the negative hedgehog regulator protein patched homolog 1 (PTCH1) and the transcriptional activator GLI1, which further potentiates hedgehog response (Kim et al., 2009).

Within this study, we evaluated the effects of chondrocyte expansion on primary cilia and determined the consequences for ciliary function, with a focus on hedgehog signalling. We showed that expansion of articular chondrocytes to passage 5 (P5) resulted in a reduction in cilia prevalence and length, with increasing passage. We showed that this was associated with reduced responsiveness of these cells to exogenous Indian hedgehog. Then, we examined the potential of drug targeting primary cilia, cytochalasin D (cyto D) and lithium chloride ( $\mathrm{LiCl})$, to restore hedgehog signalling in P5 cells. Our results highlighted the importance of maintaining ciliation in order to ensure the success of hedgehogdependent re-differentiation strategies. Moreover, the role of primary cilia in a range of growth factor signalling pathways suggested that maintaining cilia could also improve existing tissue engineering techniques.

\section{Materials and methods}

\section{Chondrocyte isolation and culture}

Primary bovine articular chondrocytes were isolated from full-depth slices of articular cartilage removed from the proximal surface of the metacarpal 
phalangeal joint. Cells were isolated by enzymatic digestion: cartilage was first incubated for $1 \mathrm{~h}$ at $37^{\circ} \mathrm{C}$ with $35 \mathrm{U} / \mathrm{mL}$ protease (P5417, Sigma-Aldrich, Poole, UK), followed by $16 \mathrm{~h}$ at $37{ }^{\circ} \mathrm{C}$ in $100 \mathrm{U} /$ $\mathrm{mL}$ collagenase (C7657, Sigma-Aldrich). Next, the cartilage digest was filtered and cells collected by centrifugation. Chondrocytes were cultured at $37{ }^{\circ} \mathrm{C}$ and $5 \% \mathrm{CO}_{2}$ in Dulbecco's modified Eagle medium (DMEM) supplemented with $10 \%$ (v/v) foetal calf serum, $1.9 \mathrm{mM}$ L-glutamine, $96 \mathrm{U} /$ $\mathrm{mL}$ penicillin, $96 \mathrm{mg} / \mathrm{mL}$ streptomycin, $19 \mathrm{mM}$ 2-[4-(2-hydroxyethyl)piperazin-1-yl]ethanesulfonic acid (HEPES) buffer and $0.74 \mathrm{mM}$ L-ascorbic acid (all from Sigma-Aldrich).

Primary chondrocytes were cultured until confluence, when they were either used for experiments as $\mathrm{P} 0$ cells or passaged for up to five passages to generate P5 cells. For experiments, chondrocytes were seeded onto serum-coated glass coverslips, plastic tissue culture plates or collagen type I-coated Flexcell ${ }^{\circledR}$ membranes (Dunn Labortechnik GMbH, Asbach, Germany) and cultured until confluence.

\section{Application of cyclic tensile strain}

To induce physiologically relevant primary cilia disassembly, chondrocytes were subjected to uniform, equibiaxial cyclic tensile strain (CTS) using the Flexcell ${ }^{\circledR}$ FX4000-T system (Dunn Labortechnik $\mathrm{GMbH}$ ) with circular loading posts with a diameter of $25 \mathrm{~mm}$. Cells were subjected to $10 \%$ strain for $24 \mathrm{~h}$ at $0.33 \mathrm{~Hz}$. For unstrained controls, chondrocytes were cultured in an identical manner, but without the application of strain. At the end of the strain period cells were immediately processed for subsequent analysis.

\section{Immunocytochemistry, confocal and super resolution microscopy}

For immunocytochemistry, samples were fixed for $10 \mathrm{~min}$ in $4 \%$ paraformaldehyde. For basal body staining, fixation was followed by $5 \mathrm{~min}$ in ice-cold methanol. Then, samples were permeabilised with $0.5 \%$ Triton X-100 and blocked with $5 \%$ donkey serum. Labelling with primary antibodies was performed overnight at $4{ }^{\circ} \mathrm{C}$. The primary antibodies used were $\alpha$-tubulin (ab4074, Abcam, Cambridge, UK), acetylated $\alpha$-tubulin (T7451, Sigma-Aldrich), ADP-ribosylation factor-like protein 13B (ARL13B) (17711-1-AP, Protein Tech, Manchester, UK), Ki67 (9129S, Cell Signalling Technology, Danvers, MA, USA) and $\gamma$-tubulin (sc-7396, Santa Cruz Biotechnology, Dallas, TX, USA), while actin was labelled with Alexa Fluor 633-conjugated phalloidin (Life Technologies, Paisley, UK). Following repeated washing, samples were incubated with Alexa Fluorconjugated secondary antibodies (Life Technologies) for $1 \mathrm{~h}$ at room temperature. Nuclei were detected with $1 \mu \mathrm{g} / \mathrm{mL}$ DAPI. Coverslips were mounted with ProLong Diamond reagent (Life Technologies).
Confocal microscopy and super resolution structured illumination microscopy (SIM) were both performed using a Zeiss 710 ELYRA PS.1 microscope (Carl Zeiss, Oberkochen, Germany) with a $63 \times / 1.4$ NA objective, yielding images with an $x-y$ pixel size of $0.1 \mu \mathrm{m}$ and $0.033 \mu \mathrm{m}$, respectively. Confocal z-stacks were generated throughout the entire cellular profile using a z-step size of $0.5 \mu \mathrm{m}$. SIM z-stacks were produced through individual cilia and processed using Zeiss Zen Black software (Carl Zeiss). In both cases, Z-stacks were reconstructed and an $x-y$ maximum intensity projection was used for the measurement of cilia length with ImageJ software (Image Processing and Analysis in Java; U.S. National Institutes of Health, Bethesda, MD, USA). Semiautomated analysis of SIM staining intensity along the entire length of cilia axonemes was performed using an ImageJ plugin, specially developed and validated in association with Dr Novak (Institute of Bioengineering, Queen Mary University of London, London, UK). For the quantification of cell proliferation, nuclear intensity of Ki-67 was quantified from confocal z-stacks using Image J and the number of positive nuclei quantified above a set threshold. The proportion of Ki-67 positive cells was expressed relative to total cell number and quantified using DAPI staining.

\section{Protein isolation and western blotting}

For protein isolation, chondrocytes were lysed in radioimmunoprecipitation assay (RIPA) buffer (Sigma-Aldrich) containing protease inhibitor cocktail (Roche, Basel, Switzerland). Following homogenisation, the lysate was centrifuged for $15 \mathrm{~min}$ at 13,000 rpm and the supernatant was collected for subsequent analysis. Protein concentration was determined by bicinchoninic acid assay. Sodium dodecyl sulphate polyacrylamide gel electrophoresis (SDS-PAGE) was performed using $20 \mu \mathrm{g}$ of total protein and 4-20\% gradient gels (Bio-Rad, Hercules, CA, USA). Proteins were transferred to nitrocellulose membranes, which were subsequently blocked with Odyssey blocking buffer (Li-Cor Biosciences, Lincoln, NE, USA) and incubated with primary antibodies overnight at $4{ }^{\circ} \mathrm{C}$. Additional primary antibodies used were Gli3 (sc-6155, Santa Cruz Biotechnology) and $\beta$-actin (ab8226, Abcam). Next, membranes were washed, incubated for $1 \mathrm{~h}$ at room temperature with appropriate infrared secondary antibodies (Li-Cor) and visualised using the Li-Cor Odyssey imaging system (Li-Cor). Analysis of band intensity was performed using Li-Cor Image Studio ${ }^{\mathrm{TM}}$ Lite.

\section{RNA isolation, cDNA synthesis and quantitative real time PCR}

RNA isolation was performed using an RNeasy Kit (Qiagen, Germantown, MD, USA) and converted to cDNA using the QuantiTect reverse transcription kit (Qiagen), according to the manufacturer's instructions. Quantitative real time PCR was 
Table 1. Primers used for real time PCR.

\begin{tabular}{|l|l|l|}
\hline Gene & Forward primer $\left(\mathbf{5}^{\prime} \mathbf{- 3}^{\prime} \mathbf{)}\right.$ & Reverse primer $\left(\mathbf{5}^{\prime} \mathbf{}^{\prime} \mathbf{3}^{\prime}\right)$ \\
\hline $18 \mathrm{~S}$ & CGGCTACCACATCCAAGGAA & GGGCCTCGAAAGAGTCCTGT \\
\hline ACAN & GAGTTTGTCAACAACAATGCC & TGGTAATTACATGGGACATCG \\
\hline COL2A & ACGTCCAGATGACCTTCCTG & GGATGAGCAGAGCCTTCTTG \\
\hline GLI1 & TTCCTCAGTGGAACCCAAG & CCTGCATTTCCAGTCATTC \\
\hline PTCH1 & ATGTCTCGCACATCAACTGG & TCGTGGTAAAGGAAAGCACC \\
\hline
\end{tabular}

performed using QuantStudio 7 (Applied Biosystems, Foster City, CA, USA) and analysed using the relative standard curve method (Larionov et al., 2005). Reactions were performed in $5 \mu \mathrm{L}$ volumes, containing $1 \mu \mathrm{L}$ cDNA (diluted 1 : 2), $2.5 \mu \mathrm{L}$ Kapa SYBR Fast Universal 2× qPCR Master Mix (Kapa Biosystems, London, UK), containing SYBR green and ROX reference dye, and $500 \mathrm{nM}$ optimised primer pairs (Table 1). Samples and standards were run in triplicate and gene expression was normalised to $18 \mathrm{~S}$ ribosomal RNA endogenous control.

\section{Statistical analysis}

Statistical analyses were performed using Graph Pad Prism 7.01 (GraphPad, La Jolla, CA, USA). When data sets adhered to normal distribution, two sample $t$-test or ANOVA with Sidaks test for multiple comparisons were used. For non-parametric data sets, MannWhitney tests were used. For contingency data, chisquared analysis was performed. $N$ referred to the number of donors and $n$ referred to the number of technical replicates analysed per experimental group. Data were presented as mean \pm SEM, except for cilia length, for which median values were described with error bars depicting 10-90 percentiles, due to the nonGaussian distributions. Details of specific statistical tests and $n$ values can be found in the figure legends.

\section{Results}

Expansion of articular chondrocytes resulted in dedifferentiation and loss of phenotype

Freshly-isolated bovine articular chondrocytes (P0) were cultured for $7 \mathrm{~d}$ in a $2 \mathrm{D}$ monolayer until confluence, then passaged again until confluence (2-3 d), for up to 5 passages (P5). Serial passages resulted in classic changes indicative of de-differentiation: very little actin stress fibre formation was observed in P0 chondrocytes, while P5 chondrocytes exhibited an increase in cell size and actin stress fibre formation and cells appeared more fibroblastic (Fig. 1A). Moreover, the expression of Sox9, the chondrocyte master transcriptional regulator required for their differentiation, was downregulated at P5 compared to P0 (Fig. 1B,C). This was accompanied by a significant reduction in the expression of the genes encoding the chondrocyte matrix proteins aggrecan $(A C A N)$ and collagen type II (COL2A) (Fig. 1D,E, respectively), thus confirming that dedifferentiation occurred in this system.

\section{Dedifferentiation of articular chondrocytes was} associated with loss of primary cilia

Primary cilia expression and structure are intrinsically linked to changes in the cytoskeleton (Pitaval et al., 2010; McMurray et al., 2013). However, there are conflicting reports as to the effect of extended cell culture upon cilia prevalence and length in other cell types (Bishop et al., 2010; Breslin et al., 2014). Therefore, primary cilia expression was examined in P0 and P5 articular chondrocytes. P0 chondrocytes exhibited primary cilia in $59 \%$ of cells, with a median length of $2.42 \mu \mathrm{m}$ (Fig. 2A,B,C). Cilia length and prevalence were significantly reduced in P5 cells, such that only $31 \%$ of cells exhibited a cilium with a median length of $2.03 \mu \mathrm{m}$ (Fig. 2A,B,C). This loss of cilia occurred progressively within each successive passage (data not shown); however, cilia loss did not occur as the result of an increased proliferation, as no significant difference in the proportion of $\mathrm{Ki}-67$ (proliferation marker)-positive cells was observed at P0 and P5 (Fig. 2D).

Tubulin acetylation promotes microtubule stability and is suggested to regulate cilia length, thus ciliary microtubules exhibit significant acetylation (Nakakura et al., 2015; Portran et al., 2017). However, despite the differences in cilium length, the level of whole cell tubulin acetylation between P0 and P5 chondrocytes, immunolabelled with $\alpha$-tubulin and acetylated $\alpha$-tubulin, did not appear different (Fig. 2E). This observation was supported by western blot analyses showing that the total level of tubulin acetylation was not significantly different between P0 and P5 (Fig. 2F,G).

\section{Dedifferentiated chondrocytes exhibited a} reduced response to exogenous hedgehog ligand The primary cilium is essential for hed gehog signalling, which promotes chondrocyte re-differentiation (Lin et al., 2014). Therefore, we examined the responsiveness of P0 and P5 chondrocytes to the addition of exogenous hedgehog ligand. Cells were treated for $24 \mathrm{~h}$ with $1 \mu \mathrm{g} / \mathrm{mL}$ recombinant Indian hedgehog (r-Ihh) and the pathway activation was examined by real time PCR. In control cells, the expression of the hedgehog markers GLI1 and PTCH1 was not significantly different in P5 cells relative to P0 (Fig. 3A,B), despite lower expression of the $83 \mathrm{kDa}$ Gli3 repressor (Gli3R, Fig. 3C) protein in P5 cells (Fig. 3D). In P0 cultures, the expression of GLI1 and PTCH1 was significantly increased by 20.97 and 6.85-fold, respectively, in response to $\mathrm{r}$-Ihh (Fig. 3A,B). By 
contrast, the expression of these genes was not significantly altered in P5 chondrocytes, consistent with the observed reduction in prevalence of cilia (Fig. 3D). The level of Gli3R was reduced relative to the untreated control in both $\mathrm{P} 0$ and $\mathrm{P} 5$ chondrocytes, following $r$-Ihh treatment (Fig. 3D). These data indicated that hedgehog pathway activation was disrupted in P5 chondrocytes, where ciliation was reduced, as a result of serial passage.

Previous studies report that the addition of hedgehog proteins to de-differentiated chondrocytes can trigger re-differentiation of these cells and induce the expression of ACAN and COL2A (Lin et al., 2014). However, in P5 cells, we found that the expression of these genes was not significantly different from the control. In contrast, $A C A N$ and COL2A expression were increased in response to $\mathrm{r}$-Ihh in $\mathrm{P} 0$ cells (data not shown).

Loss of hedgehog signalling was associated with changes in Arl13b cilia localisation

The small GTPase, Arl13b, is required for the dynamic translocation of hedgehog pathway components into and out of the cilium in response to ligand (Larkins et al., 2011). Previously, we have shown that a loss of hedgehog signalling associated with the deregulation of ciliary length is accompanied by changes in the ciliary distribution of Arl13b (Thompson et al., 2016; Thorpe et al., 2017). Therefore, we examined ciliary Arl13b content in P0 and P5 cells using SIM (Fig. 3E). Analysis of Arl13b intensity along the length of the cilium revealed that in P0 cells the localisation of Arl13b was approximately constant along the majority of the axoneme, but reduced sharply at the tip. By contrast in P5 cells, Arl13b localisation was much less even and reduced along the length of the cilium, such that intensity values in the central portion of the cilium were significantly lower in P5 cells compared to P0 (Fig. 3F). This resulted in an overall reduction in the total intensity of Arl13b within the cilium (Fig. 3F) in addition to the loss of cilia at P5 (Fig. 2A-D).

\section{Mechanically-induced reduction of primary} cilia length and prevalence in P0 chondrocytes inhibited ligand-induced hedgehog signalling Previously, we have reported that mechanical loading inhibits basal hedgehog signalling through the modulation of primary cilia length (Thompson et al., 2014). To further examine the effect that decreased cilia length and prevalence had upon ligand-induced hedgehog signalling, P0 chondrocytes were subjected to $10 \%$ CTS to reduce ciliation in combination with hedgehog stimulation. Primary cilia length was
A

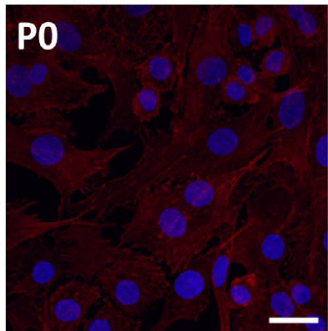

Phalloidin/DAPI

B

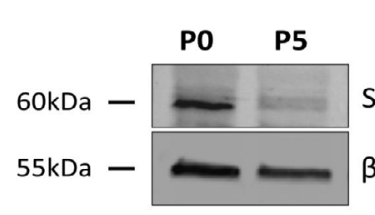

Sox9
$\beta$-tub

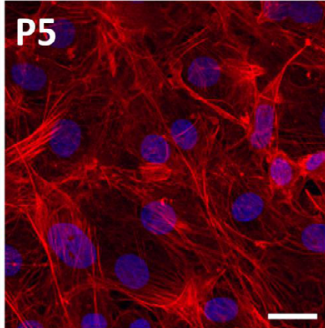

C

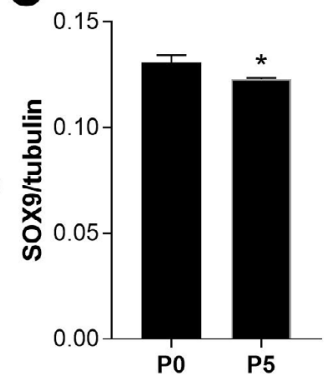

D

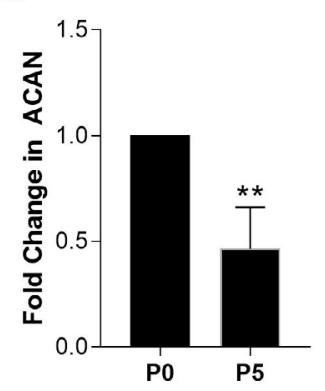

E

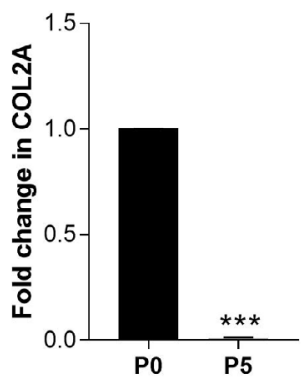

Fig. 1. Serial passage was associated with chondrocyte dedifferentiation. Primary bovine articular chondrocytes cultured in 2D monolayer at P0 and P5. (A) Representative images of actin stress fibres (phalloidin, red) counterstained with DAPI (blue). Scale bar $=20 \mu \mathrm{m}$. (B) Representative western blot of Sox 9 protein expression in $\mathrm{P} 0$ and P5 cells; $\beta$-tubulin ( $\beta$-tub) was used as a loading control. (C) Quantification of band intensity of Sox9 western blotting expressed relative to tubulin $\left(N=3, n=3\right.$, two sample $t$-test: $\left.{ }^{*} p<0.05\right)$. Realtime PCR of (D) ACAN and (E) COL2A expression; data were expressed as a fold change relative to P0 $\left(N=6, n \geq 15\right.$, paired $t$-test: ${ }^{*} p<0.05,{ }^{* *} p<0.01$ and $\left.{ }^{* *} p<0.001\right)$. 
A

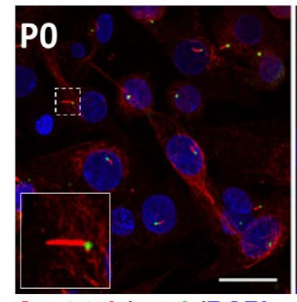

Acet-tub/ $\gamma$-tub/DAPI

E

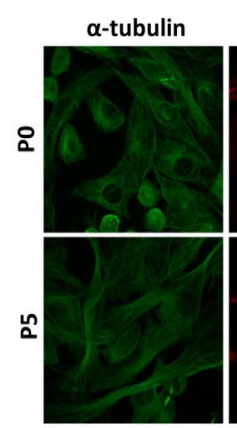

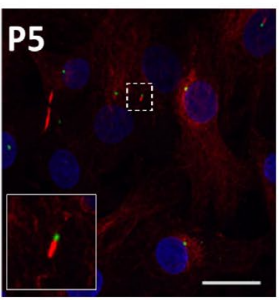

Acet-tub

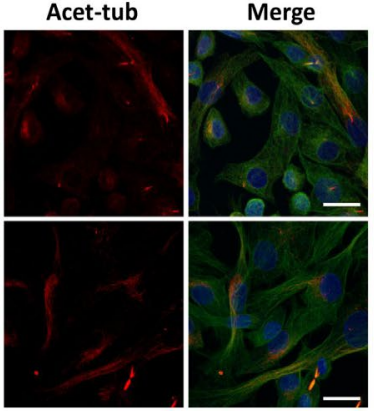

B

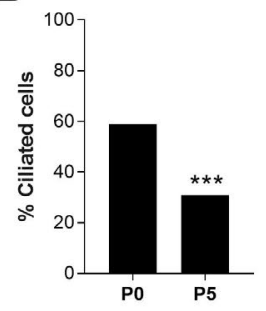

F

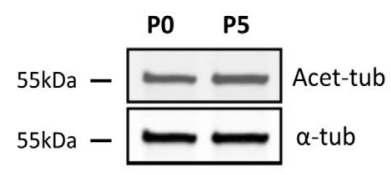

C

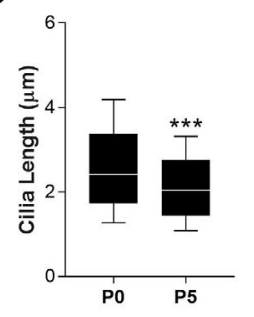

G

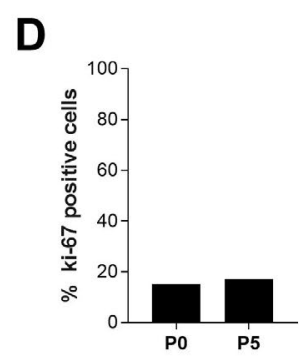

Fig. 2. Chondrocyte dedifferentiation was associated with primary cilia loss, without changes in proliferation or tubulin acetylation. Primary bovine articular chondrocytes cultured in 2D monolayer at P0 and P5. (A) Immunocytochemistry of primary cilia. Ciliary axonemes were labelled with acetylated $\alpha$-tubulin (acettub, red) and basal bodies with $\gamma$-tubulin ( $\gamma$-tub, green), nuclei were counter stained with DAPI (blue). Scale bar $=20 \mu \mathrm{m}$. Inserts showed individual cilia highlighted in the main image by the dashed white box. Quantification of $(\mathbf{B})$ cilia prevalence $\left(N=6, n \geq 529\right.$ cells, chi-squared test: $\left.{ }^{* * *} p<0.001\right)$, (C) cilia length $\left(N=6, n>135\right.$ cilia, Mann-Whitney test: $\left.{ }^{* * *} p<0.001\right)$ and (D) Ki-67 prevalence $(N=3, n \geq 187$ cells, chi-squared test). (E) Immunocytochemistry of $\alpha$-tubulin (green) and acetylated $\alpha$-tubulin (acet-tub, red), nuclei were counter stained with DAPI (blue). Scale bar $=20 \mu \mathrm{m}$. (F) Representative western blot of whole cell $\alpha$-tubulin ( $\alpha$-tub) and acetylated $\alpha$-tubulin (acet-tub) protein expression in P0 and P5 chondrocytes. (G). Quantification of band intensity of acetylated $\alpha$-tubulin (acet-tub) expressed relative to $\alpha$-tubulin ( $\alpha$-tub) $(N=3, n=3$, two sample $t$-test).

reduced from $3.31 \mu \mathrm{m}$ to $2.77 \mu \mathrm{m}$ in loaded CTS cultures (Fig. 4A,B), while cilia prevalence was reduced from $70 \%$ to $59 \%$ (Fig. 4C). The expression of GLI1 and PTCH1 was significantly upregulated in response to $r$-Ihh in the unloaded No CTS control group (Fig. 4D,E). By contrast, in the mechanicallyloaded CTS group the expression of these genes was no longer significantly upregulated by r-Ihh (Fig. 4D,E). In P5 cells, which already had reduced cilia expression, mechanical loading did not further influence ciliation or hedgehog response (Fig. 4A-C,F,G).

Actin depolymerisation increased cilia prevalence and length in P5 chondrocytes, but could not restore hedgehog signalling

Given the increased stress fibre formation in P5 chondrocytes (Fig. 1A), we examined the effects of actin depolymerisation on primary cilia. Cytochalasin D (cyto D, $10 \mu \mathrm{m}$ ) had little effect on the minimal actin stress fibre formation at P0 (Fig. 5A). Similarly, cyto D produced minimal changes in the proportion of ciliated cells in P0 cultures and had no significant effect on cilia length (Fig. 5B,C). By contrast, the enhanced formation of actin stress fibres in P5 cultures was disrupted by addition of cyto D (Fig.
5A). This was associated with a significant increase in both cilia prevalence and length, such that, with a value of $77.5 \%$, the level of ciliation exceeded that in P0 cultures, where only $53.8 \%$ of the cells were ciliated (Fig. 5B). Moreover, median cilia length was significantly increased to $4.27 \mu \mathrm{m}$ (Fig. 5C). These data suggested that the loss of primary cilia, as the result of serial passage, was likely due to changes in actin organisation.

Due to the increased ciliation in P5 cultures, as a result of cyto D treatment, we hypothesised that hedgehog responsiveness would be restored in these cells. Therefore, cultures were treated for $24 \mathrm{~h}$ with $\mathrm{r}$-Ihh in the presence of cyto D and hedgehog signalling examined by real time PCR (Fig. 5D,E). In P0 cultures, consistent with our previous data, the expression of GLI1 and PTCH1 was significantly increased by r-Ihh treatment. However, cyto D treatment inhibited this response (Fig. 5D,E) without modulating cilia expression (Fig. 5B,C). In P5 cultures, GLI1 and PTCH1 expression were not significantly increased by $\mathrm{r}$-Ihh treatment and cyto D treatment had no effect on this response (Fig. 5D,E). Thus, despite its effects on cilia at P5, cyto D was unable to restore hedgehog signalling in these expanded cells. These data suggested that even mild 
disruption of actin may have a negative effect on ciliamediated signalling, independent of changes in cilia structure or prevalence.

\section{Pharmacological modulation of cilia length partially restored hedgehog signalling}

Finally, we attempted to restore cilia in P5 cells without influencing stress fibre organisation, using the psychoactive drug lithium chloride $(\mathrm{LiCl}) . \mathrm{LiCl}$ is a potent regulator of cilia length promoting a dosedependent cilia elongation in numerous cell types, including chondrocytes (Miyoshi et al., 2009; Ou et al., 2009; Thompson et al., 2016). P0 and P5 cultures were treated with $25 \mathrm{mM} \mathrm{LiCl}$ for $24 \mathrm{~h}$ and the actin cytoskeleton examined by immunocytochemistry. Surprisingly, $\mathrm{LiCl}$ triggered some aggregation of actin structures in P0 cells, but had no significant effect on stress fibre formation in P5 cells (Fig. 5A). In $\mathrm{P} 0$ cultures, while $\mathrm{LiCl}$ did not significantly affect cilia prevalence (Fig. 5B), a dramatic increase in cilia length was observed, such that median cilia length was increased from $2.27 \mu \mathrm{m}$ to $3.48 \mu \mathrm{m}$ (Fig. 5C). In $\mathrm{P} 5$ cultures, $\mathrm{LiCl}$ resulted in a significant increase in primary cilia prevalence from $23.9 \%$ to $52.8 \%$ (Fig. 5B), which was accompanied by an increase in cilia length from $2.00 \mu \mathrm{m}$ to $2.45 \mu \mathrm{m}$ (Fig. 5C). Thus, $\mathrm{LiCl}$ restored cilia length and prevalence in P5 cells, such that these values were no longer significantly different to P0 cells (Fig. 5B,C).

Consistent with a previous report (Thompson et al., 2016), the induction of GLI1 and PTCH1 in response to $\mathrm{r}$-Ihh was inhibited by $\mathrm{LiCl}$ in $\mathrm{P0}$ cells (Fig. 5D,E).
A

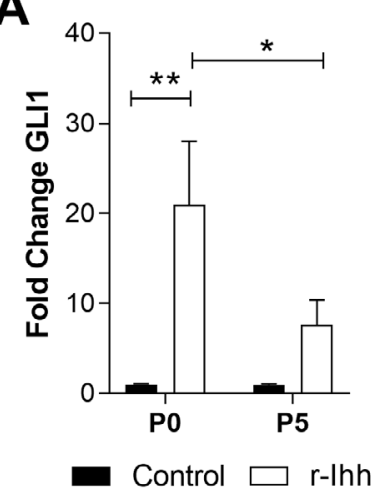

B

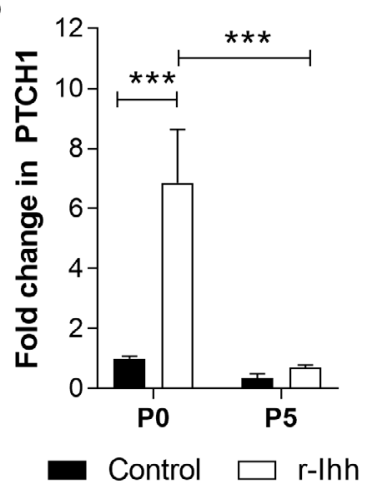

C

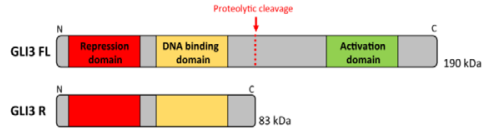

D

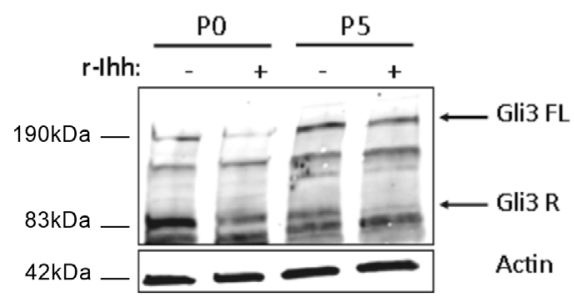

E

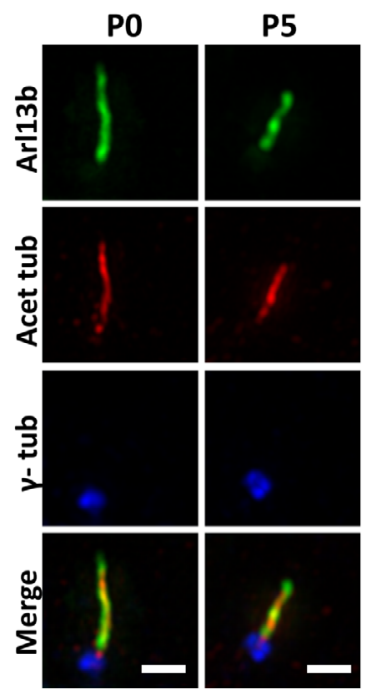

$F$

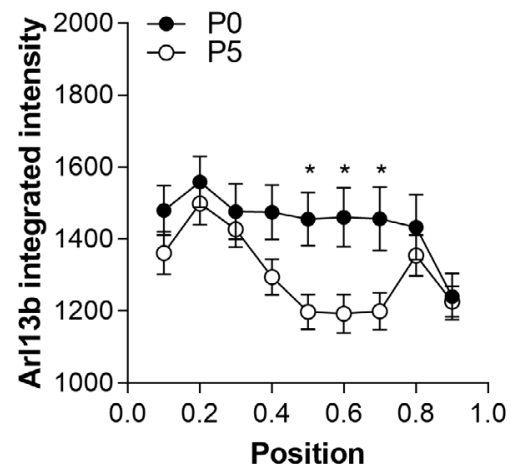

G

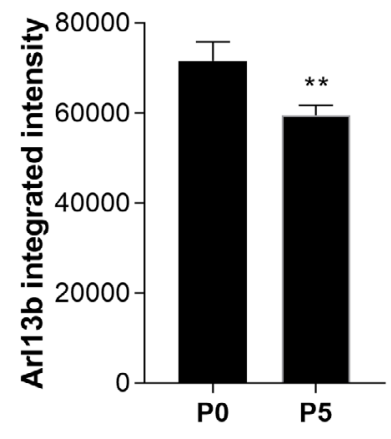

Fig. 3. Hedgehog pathway activation was disrupted in chondrocytes following serial passage, in association with changes in cilia expression and Arl13b cilia localisation. P0 and P5 chondrocytes cultured in 2D monolayer and treated for $24 \mathrm{~h}$ with r-Ihh. Real time PCR of (A) GLI1 and (B) PTCH1 expression (N=6, $n \geq 16$, two-way ANOVA with Sidaks multiple comparison: ${ }^{*} p<0.05$, ${ }^{* *} p<0.01$ and $\left.{ }^{* * *} p<0.001\right)$. (C) Schematic of full-length and repressor forms of Gli3 (adapted from Alman, 2015). (D) Representative western blot of Gli3 expression in P0 and P5 cells, actin was used as a loading control. (E) Structured illumination microscopy of primary cilia from P0 and P5 cells labelled for Arl13b (green), acetylated $\alpha$-tubulin (acet-tub; red) and $\gamma$-tubulin ( $\gamma$-tub; blue). Scale bar $=1 \mu \mathrm{m}$. (F) Arl13b intensity along the length of the ciliary axoneme, where position $0.0=$ cilium base and $1.0=$ ciliary tip $(N=3, n \geq 67$ cilia, two-way ANOVA with Sidaks multiple comparison: $\left.{ }^{*} p<0.05\right)$. (G) Total Arl13b integrated intensity $(N=3, n \geq 67$ cilia, Mann-Whitney test: $\left.{ }^{*} p<0.05\right)$. 
By contrast, in P5 cells, in the presence of $\mathrm{LiCl}$, the expression of GLI1 was significantly increased by the treatment with r-Ihh. The up-regulated GLI1 reached levels similar to the ones in P0 cells in the absence of $\mathrm{LiCl}$ (Fig. 5D). However, there was no significant effect on PTCH1 expression (Fig. 5E). These results suggested that $\mathrm{LiCl}$ induced a partial rescue of cilia hedgehog function in P5 cultures, associated with reversing the reduction in cilia prevalence and length induced by serial passages.

\section{Discussion}

This study examined the effects of serial passage and dedifferentiation on chondrocyte primary cilia. We demonstrated that dedifferentiation was accompanied by primary cilia loss, most likely due to changes in actin organisation. Primary cilia loss resulted in the disruption of ligand-dependent hedgehog signalling. We showed that a partial rescue of hedgehog signalling could be achieved in passaged cells through the restoration of primary cilia with $\mathrm{LiCl}$.

Our findings were consistent with previous studies performed in other cell types showing that downregulation or inhibition of hedgehog signalling occurs as consequence of dedifferentiation and cellular senescence (Bishop et al., 2010; Breslin et al., 2014). However, the mechanisms responsible for this phenomenon are still objects of debate. The cilium is absolutely required for hedgehog signalling, as many of the components of this pathway traffic through the ciliary compartment and are modified here in order to regulate pathway activity (Corbit et al., 2005; Haycraft et al., 2005). Therefore, our findings suggested that it was the loss of this regulatory compartment that resulted in pathway inhibition. Furthermore, the reduced length of cilia observed in passaged cells was indicative of IFT dysfunction. IFT is required for

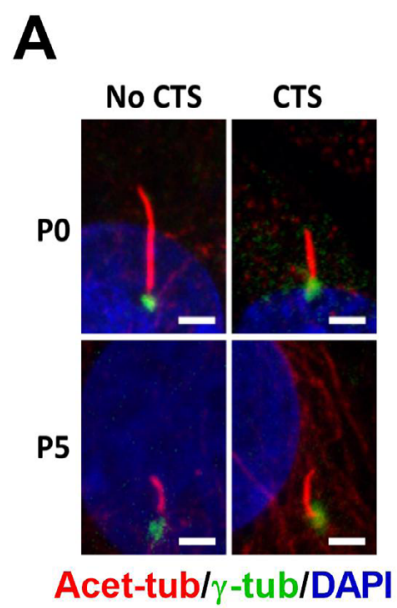

B
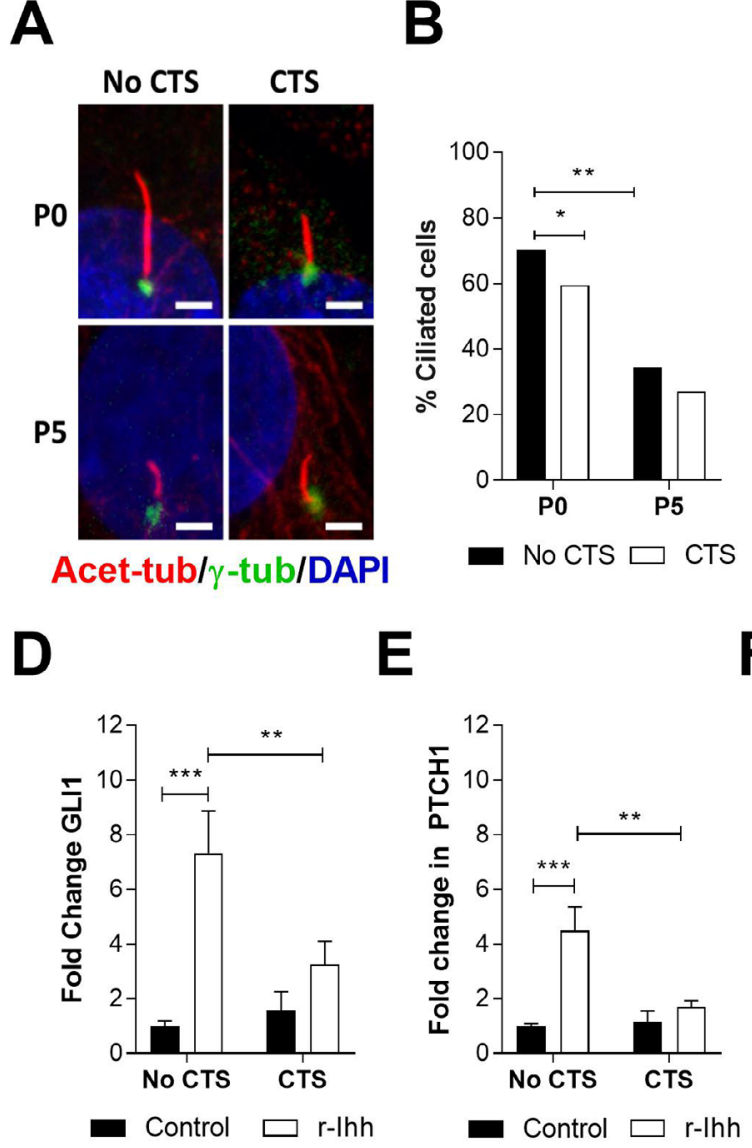

E

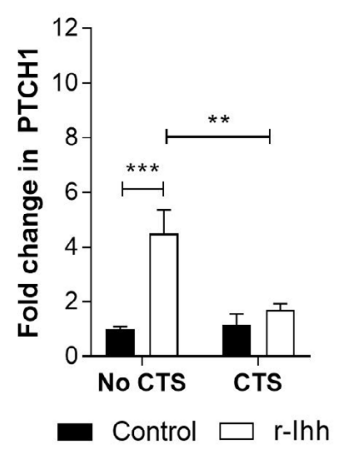

C

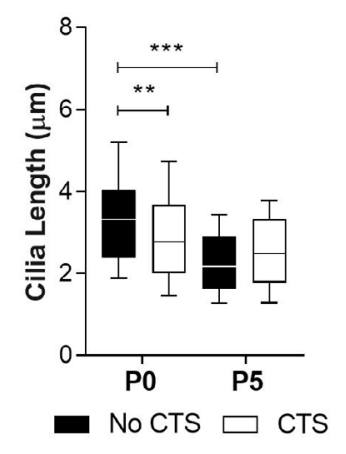

$\mathbf{F}$

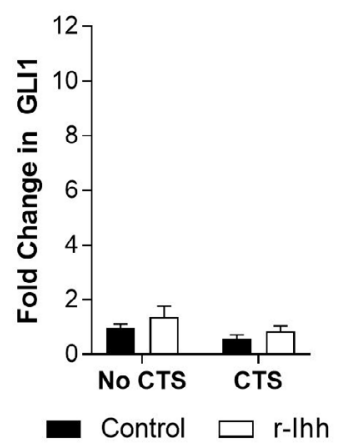

G

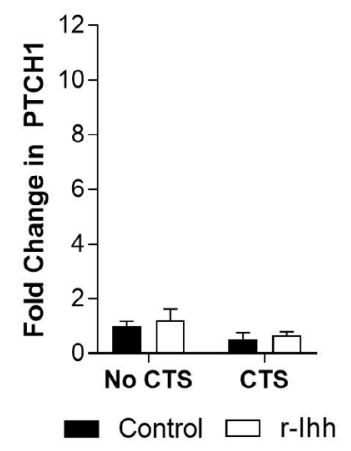

Fig. 4. Mechanical loading reduced primary cilia length and prevalence in P0 chondrocytes and inhibited ligand-induced hedgehog signalling, replicating the behaviour of P5 cells. P0 and P5 chondrocytes were subjected to $10 \mathrm{CTS}, 0.33 \mathrm{~Hz}$ for $24 \mathrm{~h}$ in the presence of $\mathrm{r}$-Ihh. (A) Immunocytochemistry of primary cilia. Ciliary axonemes were labelled with acetylated $\alpha$-tubulin (red) and basal bodies with $\gamma$-tubulin (green), nuclei were counter stained with DAPI (blue). Scale bar $=2 \mu \mathrm{m}$. Quantification of (B) primary cilia prevalence $\left(N=3, n \geq 290\right.$ cells, chi-squared test: $\left.{ }^{* *} p<0.001\right)$ and $(\mathbf{C})$ primary cilia length $(N=3$, $n \geq 165$ cilia, Mann- Whitney test: ${ }^{* *} p<0.01$ ). Real time PCR of (D) GLI1 and (E) PTCH1 expression in P0 cells and (E) GLI1 and (F) PTCH1 in P5 cells. Data were normalised to GAPDH and expressed as a fold change relative to the NO CTS control $(N=3, n \geq 8$, two-way ANOVA with Sidaks multiple comparison: ** $p<0.01$ and $\left.{ }^{* * *} p<0.001\right)$. 
cilia functionality in addition to cilia construction and maintenance; therefore, we would expect a reduced axoneme length to have direct consequences on signalling. Indeed, increasing numbers of studies are reporting small changes in the size of the ciliary compartment accompanied by more dramatic effects upon signalling (Tran et al., 2008; Lancaster et al., 2011; McMurray et al., 2013; Thompson et al., 2014; Sherpa et al., 2016; Snouffer et al., 2017; Spasic and Jacobs 2017).

Previously, we have shown that alterations in the ciliary distribution of Arl13b occur as a result of ciliary lengthening and are associated with hedgehog inhibition (Thompson et al., 2016). While this previous study reported a reduction in Arl13b localisation to the ciliary tip, in the current study
A
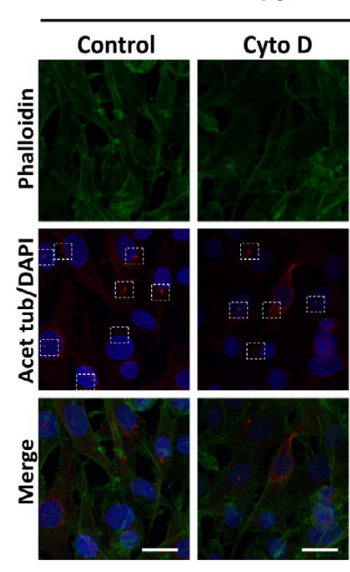

B

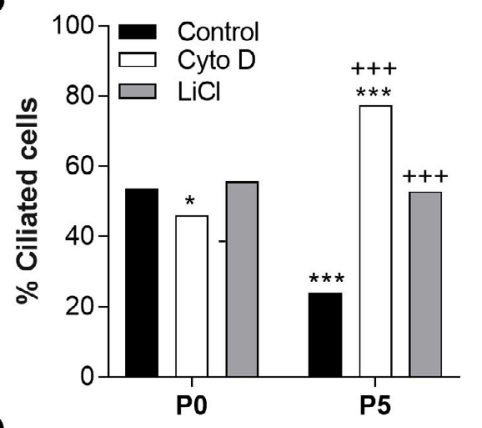

D

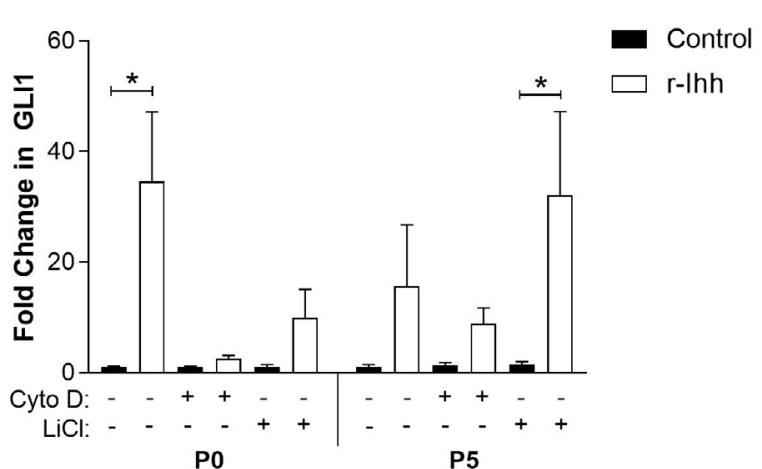

E

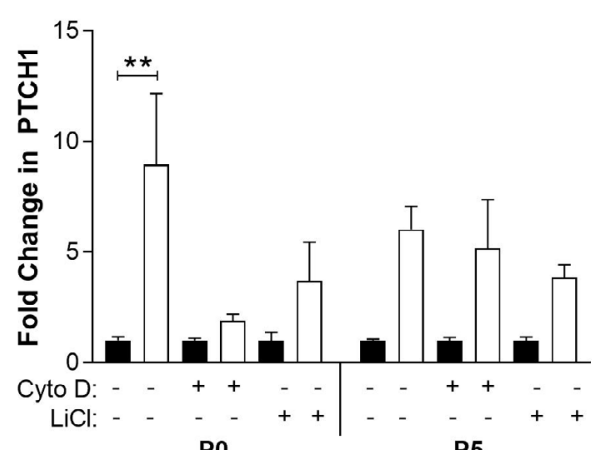

P0

P5

C
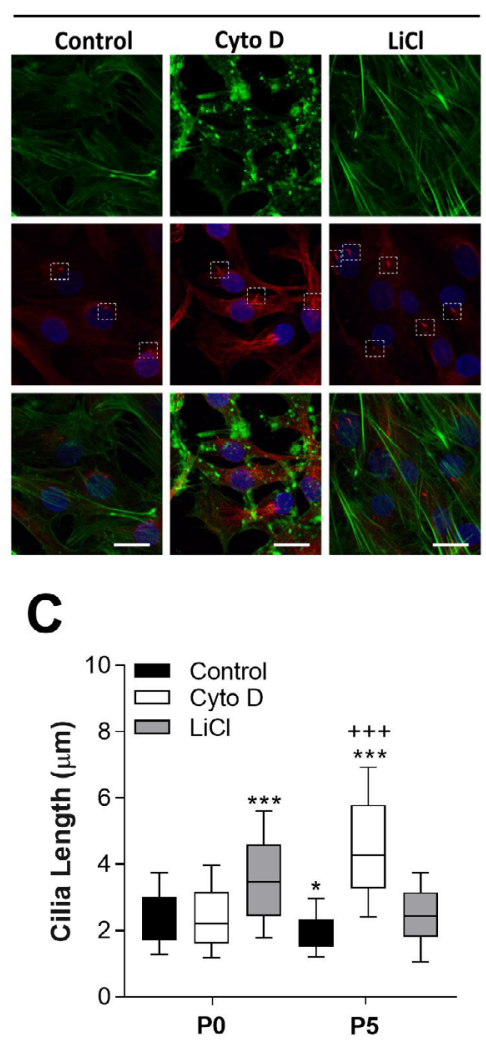

Fig. 5. LiCl increased primary cilia expression in P5 cells and partially restored hedgehog signalling. Primary bovine articular chondrocytes cultured in 2D monolayer at P0 and P5 then treated for $24 \mathrm{~h}$ with cytochalasin $\mathrm{D}$ (cyto D) and lithium chloride ( $\mathrm{LiCl})$ \pm recombinant Indian hedgehog (r-Ihh). (A) Immunocytochemistry of actin stress fibres (phalloidin, green) and acetylated $\alpha$-tubulin (acet-tub; red), nuclei were counter stained with DAPI (blue). Scale bar $=20 \mu \mathrm{m}$. Individual cilia were highlighted by dashed white boxes. Quantification of (B) cilia prevalence $(N=3, n \geq 132$ cells, chi-squared test: ${ }^{* * *} p<0.05$ and $\left.{ }^{* * *} p<0.001\right)$ and (C) cilia length $(N=3, n \geq 50$ cilia, Mann-Whitney test: ${ }^{*} p<0.05$ and ${ }^{* * *} p<0.001$ relative to $\mathrm{P} 0$ control, $+++p<0.001$ relative to P5 control). Real time PCR of (D) GLI1 and (E) PTCH1 expression, data were expressed as a fold change relative to the respective control for each condition $(N=3, n \geq 6$, twoway ANOVA with Sidaks multiple comparison: $\left.{ }^{*} p<0.05,{ }^{* *} p<0.01\right)$. 
we observed a reduction in Arl13b along the mid region of the axoneme, which accompanied ciliary shortening in P5 cultures (Fig. 3D,E, F). While this may also be indicative of alterations in trafficking, production of Gli3R was still observed in P5 cultures, albeit at a reduced level. Moreover, Gli3R expression was reduced following ligand treatment (Fig. 3C). The production of Gli3R is dependent on the trafficking of the full-length protein through the ciliary compartment, where it is modified by PKA and targeted for proteasomal cleavage (Sasaki et al., 1999; Huangfu et al., 2003). Thus, these data suggested that ciliary trafficking was intact in P5 cilia and supported the hypothesis that reduced Gli3R production was more likely a consequence of cilia loss.

Mechanical loading triggers primary cilia disassembly in a histone deacetylase 6 (HDAC6)dependent manner (McGlashan et al., 2010; Thompson et al., 2014). We utilised this observation to reduce cilia length and prevalence in P0 cultures. Despite a comparatively small reduction in ciliation (Fig. 3A, B,C), ligand-dependent hedgehog signalling was inhibited by loading, providing further support for this hypothesis. However, the complete inhibition of this response suggested that there might be additional mechanisms at play. Primary cilia have been implicated in chondrocyte mechanosignalling and regulation of $A C A N$ expression in response to load (McGlashan et al., 2007; Wann et al., 2012). While the effects of cilia loss on mechanosignalling were not investigated in the current study, future studies should examine the influence of cilia restoration upon this pathway, as effective matrix production is a key factor for the successful engineering of cartilage tissue. Indeed, a recent study by Spasic and Jacobs (2017) reports that targeting cilia with $\mathrm{LiCl}$ can improve mechanosensitivity in bone cells through its effects on primary cilia.

Several actin regulatory molecules are known to influence cilia length (Kim et al., 2010), while the presence of branched F-actin negatively regulates ciliogenesis (for review see Yan and Zhu, 2013). Thus, the reduction in cilia expression observed in this study was likely a consequence of increased stress fibre formation and alterations in actin cytoskeletal tension that accompany dedifferentiation [(Benya, 1988; Parreno et al., 2017) ; Fig. 1]. In support of this hypothesis, we found that cyto D-induced depolymerisation of the actin cytoskeleton in $\mathrm{P} 5$ cells restored ciliation beyond P0 levels (Fig. 5A,B,C). Interestingly, in the current study, we observed a higher level of ciliation and increased cilia length on Flexcell ${ }^{\circledR}$ membranes relative to the stiffer glass coverslips (Fig. 2 and 4). This suggested that the mechanical environment modulated chondrocyte ciliation, possibly through associated changes in actin organisation. Such findings have implications for the expansion of chondrocytes and the design of 3D scaffold materials for cartilage tissue engineering.

Restoration of primary cilia in P5 chondrocytes through actin depolymerisation could not restore hedgehog response (Fig. 5). Moreover, cyto D treatment inhibited hedgehog signalling in P0 chondrocytes (Fig. 5). Together these data suggested that a complex relationship existed between actin, cilia and hedgehog. In addition to the cilia-dependent regulation of hedgehog signalling (Bershteyn et al., 2010), the actin cytoskeleton can regulate hedgehog signalling through cilia-independent mechanisms. The dual specificity tyrosine-phosphorylationregulated kinase 1A (DYRKA1) inhibits endogenous hedgehog signalling by negatively regulating the actin stabilising actin-binding LIM (ABLIM) proteins (Schneider et al., 2015). Phosphorylation of ABLIM1 reduces F-actin assembly and nuclear localisation of the megakaryoblastic leukemia 1 (MKL1) protein. This reduces activation of the Jumonji domain-containing histone demethylase 1A (JMJD1A, encoded by the Kdm3a gene), which, in turn, stimulates the transcriptional activity of Gli1 (Schneider et al., 2015), possibly accounting for hedgehog disruption in P0 cells. However, $k d m 3 a$ mutant mice exhibit phenotypes overlapping with mouse models of ciliopathies (Inagaki et al., 2009) and a recent study from Yeyati et al. (2017) demonstrates that KDM3A (JMJD1A) plays a role in mammalian ciliogenesis through the regulation of actin dynamics. Thus, separating cilia-dependent and -independent effects of actin modulation on hedgehog signalling is complex.

In this study, we employed $\mathrm{LiCl}$ to modify ciliation in P5 cells. This drug is widely used to consistently promote cilia elongation across numerous cell types, in contrast to more targeted molecular approaches (Miyoshi et al., 2009; Ou et al., 2009, Thompson et al., 2016). Stress fibre formation was still apparent in P5 cultures following $\mathrm{LiCl}$ treatment, which restored ciliation and cilia length to the levels of P0 cells. However, only a partial rescue of Hh signalling was achieved (Fig. 5D,E). The shedding of ciliary GPCRs, such as Gpr161, within ciliary ectosomes, is regulated by actin and can regulate hedgehog signalling (Nager et al., 2017). Ciliary ectosomes are shed from the ciliary tip as a mean for removing activated signalling molecules from cilia, which is accompanied by significant loss of ciliary material and ciliary shortening (Nager et al., 2017). Therefore, if cilia loss in P5 chondrocytes occurred in part as a result of ectosome shedding, then it is conceivable that while $\mathrm{LiCl}$ might have effectively restored the cilia structure, the previous loss of protein by ectosomes might prevent full recovery of hedgehog signalling.

Hedgehog ligands can promote cartilage matrix production and the re-differentiation of articular chondrocytes (Kellner et al., 2002; Lin et al., 2014). Therefore, restoration of this signalling pathway could be beneficial for the generation of tissue engineered constructs. Indeed, Kellner et al. (2002) reports increased matrix production in tissue engineered constructs in the presence of hedgehog. This response is enhanced when constructs are 
cultured in low serum conditions (Kellner et al., 2002), which are likely to increase cilia expression (Wheatley et al., 1996). Thus, this study further supports our finding that cilia restoration in dedifferentiated cells could improve hedgehog responsiveness. Lin et al. (2014) report that, in mice, hedgehog ligands induce the expression of ACAN, COL2 and SOX9 in P5 chondrocytes and improve the integration of tissue engineered constructs into cartilage defects. However, in the current study, while r-Ihh increased $A C A N$ and COL2 expression in P0 cultures, it did not induce the expression of matrix genes in P5 cells. This was consistent with cells at P5 showing cilia loss (Fig. 2) and disruption of hedgehog signalling (Fig. 3A-C and data not shown). However, the partial rescue of hedgehog signalling achieved with $\mathrm{LiCl}$ at P5 was not sufficient to restore this downstream response in terms of cartilage matrix gene expression (data not shown).

Targeting cilia for tissue engineering procedures and the concept of ciliotherapy has been in existence for some time with several studies providing proof of concept data suggesting that targeting this organelle can have great therapeutic benefit. For example, the restoration of cilia in pancreatic cancer reduces tumour size and disease progression (Gradilone et al., 2013). While in the kidney, lengthening of primary cilia with feneldopam can effectively increase serum nitric oxide and reduce blood pressure in a mouse model of polycystic kidney disease (Kathem et al., 2014). Therefore, it is timely that this study assessed the implications of cell expansion on cilia structurefunction, such that future tissue engineering strategies may consider targeting this organelle for effective cartilage production.

\section{Conclusions}

Maintaining chondrocyte phenotype is a necessary part of cartilage tissue engineering. In the present study, we showed for the first time, that chondrocyte expansion and dedifferentiation disrupted primary cilia expression and hedgehog signalling and that this was associated with actin reorganisation. This excluded the use of hedgehog ligands for re-differentiation of expanded chondrocytes. However, treatment with $\mathrm{LiCl}$ partially restored cilia expression and hedgehog responsiveness, thus future strategies for tissue engineering cartilage should consider targeting primary cilia in order to restore normal chondrocyte function and enhance cartilage production.

\section{Acknowledgements}

This work was funded by a project grant from the UK Medical Research Council (No.: MR/L002876/1, PI: MK). In addition, James Plant was supported from an EPSRC PhD studentship from the Institute of Bioengineering at Queen Mary University of London. We greatly thank Ms Eemin Tan for conducting preliminary experiments as part of her intercalated BSc in Biomedical Engineering at Queen Mary University of London. Philip Beales is an NIHR Senior Investigator and was supported by the NIHR Great Ormond Street Hospital Biomedical Research Centre (GOSH BRC).

\section{References}

Alman BA (2015) The role of hedgehog signalling in skeletal health and disease. Nat Rev Rheumatol 11: 552-560.

Benya PD (1988) Modulation and reexpression of the chondrocyte phenotype; mediation by cell shape and microfilament modification. Pathol Immunopathol Res 7: 51-54.

Benya PD, Padilla SR, Nimni ME (1978) Independent regulation of collagen types by chondrocytes during the loss of differentiated function in culture. Cell 15: 1313-1321.

Bershteyn M, Atwood SX, Woo WM, Li M, Oro AE (2010) Mim and cortactin antagonism regulates ciliogenesis and hedgehog signaling. Dev Cell 19: 270-283.

Bishop CL, Bergin AM, Fessart D, Borgdorff V, Hatzimasoura E, Garbe JC, Stampfer MR, Koh J, Beach DH (2010) Primary cilium-dependent and -independent hedgehog signaling inhibits p16(ink4a). Mol Cell 40: 533-547.

Breslin L, Prosser SL, Cuffe S, Morrison CG (2014) Ciliary abnormalities in senescent human fibroblasts impair proliferative capacity. Cell Cycle 13: 27732779.

Christensen ST, Clement CA, Satir P, Pedersen LB (2012) Primary cilia and coordination of receptor tyrosine kinase (rtk) signalling. J Pathol 226: 172-184.

Corbit KC, Aanstad P, Singla V, Norman AR, Stainier DY, Reiter JF (2005) Vertebrate smoothened functions at the primary cilium. Nature 437: 10181021.

Gradilone SA, Radtke BN, Bogert PS, Huang BQ, Gajdos GB, LaRusso NF (2013) Hdac6 inhibition restores ciliary expression and decreases tumor growth. Cancer Res 73: 2259-2270.

Haycraft CJ, Banizs B, Aydin-Son Y, Zhang Q, Michaud EJ, Yoder BK (2005) Gli2 and gli3 localize to cilia and require the intraflagellar transport protein polaris for processing and function. PLoS Genet 1: e53.

Haycraft CJ, Serra R (2008) Cilia involvement in patterning and maintenance of the skeleton. Curr Top Dev Biol 85: 303-332.

Holtzer H, Abbott J, Lash J, Holtzer S (1960) The loss of phenotypic traits by differentiated cells in vitro, i. dedifferentiation of cartilage cells. Proc Natl Acad Sci USA 46: 1533-1542. 
Huangfu D, Liu A, Rakeman AS, Murcia NS, Niswander L, Anderson KV (2003) Hedgehog signalling in the mouse requires intraflagellar transport proteins. Nature 426: 83-87.

Inagaki T, Tachibana M, Magoori K, Kudo H, Tanaka T, Okamura M, Naito M, Kodama T, Shinkai Y, Sakai J (2009) Obesity and metabolic syndrome in histone demethylase jhdm2a-deficient mice. Genes Cells 14: 991-1001.

Kathem SH, Mohieldin AM, Abdul-Majeed S, Ismail SH, Altaei QH, Alshimmari IK, Alsaidi MM, Khammas H, Nauli AM, Joe B, Nauli SM (2014) Ciliotherapy: a novel intervention in polycystic kidney disease. J Geriatr Cardiol 11: 63-73.

Kellner K, Lang K, Papadimitriou A, Leser U, Milz S, Schulz MB, Blunk T, Gopferich A (2002) Effects of hedgehog proteins on tissue engineering of cartilage in vitro. Tissue Eng 8: 561-572.

Kim J, Kato M, Beachy PA (2009) Gli2 trafficking links hedgehog-dependent activation of smoothened in the primary cilium to transcriptional activation in the nucleus. Proc Natl Acad Sci USA 106: 21666-21671.

Kim J, Lee JE, Heynen-Genel S, Suyama E, Ono K, Lee K, Ideker T, Aza-Blanc P, Gleeson JG (2010) Functional genomic screen for modulators of ciliogenesis and cilium length. Nature 464: 1048-1051.

Ko EC, Fujihara Y, Ogasawara T, Asawa Y, Nishizawa S, Nagata S, Takato T, Hoshi K (2012) Bmp-2 embedded atelocollagen scaffold for tissueengineered cartilage cultured in the medium containing insulin and triiodothyronine-a new protocol for three-dimensional in vitro culture of human chondrocytes. Tissue Eng Part C Methods 18: 374-386.

Lancaster MA, Schroth J, Gleeson JG (2011) Subcellular spatial regulation of canonical wnt signalling at the primary cilium. Nat Cell Biol 13: 700-707.

Larionov A, Krause A, Miller W (2005) A standard curve based method for relative real time pcr data processing. BMC Bioinformatics 6: 62.

Larkins CE, Aviles GD, East MP, Kahn RA, Caspary T (2011) Arl13b regulates ciliogenesis and the dynamic localization of shh signaling proteins. Mol Biol Cell 22: 4694-4703.

Lin L, Shen Q, Xue T, Duan X, Fu X, Yu C (2014) Sonic hedgehog improves redifferentiation of dedifferentiated chondrocytes for articular cartilage repair. PLoS One 9: e88550.

Lohmann CH, Schwartz Z, Niederauer GG, Carnes DL, Jr., Dean DD, Boyan BD (2000) Pretreatment with platelet derived growth factor-bb modulates the ability of costochondral resting zone chondrocytes incorporated into pla/pga scaffolds to form new cartilage in vivo. Biomaterials 21: 49-61.

McGlashan SR, Haycraft CJ, Jensen CG, Yoder BK, Poole CA (2007) Articular cartilage and growth plate defects are associated with chondrocyte cytoskeletal abnormalities in tg737orpk mice lacking the primary cilia protein polaris. Matrix Biol 26: 234-246.
McGlashan SR, Knight MM, Chowdhury TT, Joshi P, Jensen CG, Kennedy S, Poole CA (2010) Mechanical loading modulates chondrocyte primary cilia incidence and length. Cell Biol Int 34: 441-446.

McMurray RJ, Wann AK, Thompson CL, Connelly JT, Knight MM (2013) Surface topography regulates wnt signaling through control of primary cilia structure in mesenchymal stem cells. Sci Rep 3: 3545.

Miyoshi K, Kasahara K, Miyazaki I, Asanuma M (2009) Lithium treatment elongates primary cilia in the mouse brain and in cultured cells. Biochem Biophys Res Commun 388: 757-762.

Mukhopadhyay S, Wen X, Ratti N, Loktev A, Rangell L, Scales SJ, Jackson PK (2013) The ciliary g-protein-coupled receptor gpr161 negatively regulates the sonic hedgehog pathway via camp signaling. Cell 152: 210-223.

Nager AR, Goldstein JS, Herranz-Perez V, Portran D, Ye F, Garcia-Verdugo JM, Nachury MV (2017) An actin network dispatches ciliary gpcrs into extracellular vesicles to modulate signaling. Cell 168: 252-263.

Nakakura T, Asano-Hoshino A, Suzuki T, Arisawa K, Tanaka H, Sekino Y, Kiuchi Y, Kawai K, Hagiwara $H$ (2015) The elongation of primary cilia via the acetylation of alpha-tubulin by the treatment with lithium chloride in human fibroblast kd cells. Med Mol Morphol 48: 44-53.

Ou Y, Ruan Y, Cheng M, Moser JJ, Rattner JB, van der Hoorn FA (2009) Adenylate cyclase regulates elongation of mammalian primary cilia. Exp Cell Res 315: 2802-2817.

Parreno J, Nabavi Niaki M, Andrejevic K, Jiang A, Wu PH, Kandel RA (2017) Interplay between cytoskeletal polymerization and the chondrogenic phenotype in chondrocytes passaged in monolayer culture. J Anat 230: 234-248.

Pitaval A, Tseng Q, Bornens M, Thery M (2010) Cell shape and contractility regulate ciliogenesis in cell cycle-arrested cells. J Cell Biol 191: 303-312.

Portran D, Schaedel L, Xu Z, Thery M, Nachury MV (2017) Tubulin acetylation protects long-lived microtubules against mechanical ageing. Nat Cell Biol 19: 391-398.

Praetorius HA, Spring KR (2001) Bending the mdck cell primary cilium increases intracellular calcium. J Membr Biol 184: 71-79.

Rai V, Dilisio MF, Dietz NE, Agrawal DK (2017) Recent strategies in cartilage repair: a systemic review of the scaffold development and tissue engineering. J Biomed Mater Res A 8: 2343-2354.

Sasaki H, Nishizaki Y, Hui C, Nakafuku M, Kondoh H (1999) Regulation of gli2 and gli3 activities by an amino-terminal repression domain: implication of gli2 and gli3 as primary mediators of shh signaling. Development 126: 3915-3924.

Schneider L, Clement CA, Teilmann SC, Pazour GJ, Hoffmann EK, Satir P, Christensen ST (2005) PDGFRalphaalpha signaling is regulated through the primary cilium in fibroblasts. Curr Biol 15: 1861-1866. 
Schneider P, Bayo-Fina JM, Singh R, Kumar Dhanyamraju P, Holz P, Baier A, Fendrich V, Ramaswamy A, Baumeister S, Martinez ED, Lauth M (2015) Identification of a novel actin-dependent signal transducing module allows for the targeted degradation of gli1. Nat Commun 6: 8023.

Sharma N, Kosan ZA, Stallworth JE, Berbari NF, Yoder BK (2011) Soluble levels of cytosolic tubulin regulate ciliary length control. Mol Biol Cell 22: 806816.

Sherpa RT, Atkinson KF, Ferreira VP, Nauli SM (2016) Rapamycin increases length and mechanosensory function of primary cilia in renal epithelial and vascular endothelial cells. Int Educ Res J 2: 91-97.

Snouffer A, Brown D, Lee H, Walsh J, Lupu F, Norman R, Lechtreck K, Ko HW, Eggenschwiler J (2017) Cell cycle-related kinase (ccrk) regulates ciliogenesis and hedgehog signaling in mice. PLoS Genet 13: e1006912.

Spasic M, Jacobs CR (2017) Lengthening primary cilia enhances cellular mechanosensitivity. Eur Cell Mater 33: 158-168.

Thompson CL, Chapple JP, Knight MM (2014) Primary cilia disassembly down-regulates mechanosensitive hedgehog signalling: a feedback mechanism controlling adamts-5 expression in chondrocytes. Osteoarthritis Cartilage 22: 490-498.

Thompson CL, Wiles A, Poole CA, Knight MM (2016) Lithium chloride modulates chondrocyte primary cilia and inhibits hedgehog signaling. FASEB J 30: 716-726.

Thorpe SD, Gambassi S, Thompson CL, Chandrakumar C, Santucci A, Knight MM (2017) Reduced primary cilia length and altered arl13b expression are associated with deregulated chondrocyte hedgehog signaling in alkaptonuria. J Cell Physiol 9: 2407-2417.

Tran PV, Haycraft CJ, Besschetnova TY, TurbeDoan A, Stottmann RW, Herron BJ, Chesebro AL, Qiu H, Scherz PJ, Shah JV, Yoder BK, Beier DR (2008) Thm1 negatively modulates mouse sonic hedgehog signal transduction and affects retrograde intraflagellar transport in cilia. Nat Genet 40: 403-410.

Veland IR, Awan A, Pedersen LB, Yoder BK, Christensen ST (2009) Primary cilia and signaling pathways in mammalian development, health and disease. Nephron Physiol 111: 39-53.

Wann AK, Chapple JP, Knight MM (2014) The primary cilium influences interleukin-1beta-induced nfkappab signalling by regulating ikk activity. Cell Signal 26: 1735-1742.

Wann AK, Knight MM (2012) Primary cilia elongation in response to interleukin-1 mediates the inflammatory response. Cell Mol Life Sci 69: 29672977.

Wann AK, Zuo N, Haycraft CJ, Jensen CG, Poole CA, McGlashan SR, Knight MM (2012) Primary cilia mediate mechanotransduction through control of atpinduced ca2+ signaling in compressed chondrocytes. FASEB J 26: 1663-1671.
Wheatley DN, Wang AM, Strugnell GE (1996) Expression of primary cilia in mammalian cells. Cell Biol Int 20: 73-81.

Yan X, Zhu X (2013) Branched f-actin as a negative regulator of cilia formation. Exp Cell Res 319: 147-151.

Yeyati PL, Schiller R, Mali G, Kasioulis I, Kawamura A, Adams IR, Playfoot C, Gilbert N, van Heyningen V, Wills J, von Kriegsheim A, Finch A, Sakai J, Schofield CJ, Jackson IJ, Mill P (2017) Kdm3a coordinates actin dynamics with intraflagellar transport to regulate cilia stability. J Cell Biol 216: 999-1013.

Zhu D, Shi S, Wang H, Liao K (2009) Growth arrest induces primary-cilium formation and sensitizes igf1-receptor signaling during differentiation induction of 3t3-11 preadipocytes. J Cell Sci 122: 2760-2768.

Zimber MP, Tong B, Dunkelman N, Pavelec R, Grande D, New L, Purchio AF (1995) Tgf-beta promotes the growth of bovine chondrocytes in monolayer culture and the formation of cartilage tissue on three-dimensional scaffolds. Tissue Eng 1: 289-300.

\section{Discussion with Reviewer}

Farshid Guilak: What is the relationship between continuous biaxial tensile stretch of chondrocytes and expansion-induced dedifferentiation? Please discuss how these mechanisms might be further interrelated to influence the cilia.

Authors: Mechanotransduction is involved in the maintenance of the chondrocyte phenotype, such that the application of dynamic mechanical loading can promote the chondrogenic phenotype (Holmvall et al., 1995; Wong et al., 2003). Indeed, the culture of primary bovine chondrocytes on a continuously expanding surface can inhibit the dedifferentiation that accompanies serial expansion (Rosenzweig et al., 2012). Mechanical stimuli regulate the maintenance of chondrocyte ciliary structure, which is suggested to function as a mean to modify ciliary responses to environmental cues (McGlashan et al., 2008; McGlashan et al., 2010; Thompson et al., 2014). In the current study, we reported that cells undergoing serial expansion exhibited cilia loss with an accompanying defect in cilia-mediated hedgehog signalling, suggesting that environmental regulation of ciliary signalling was disrupted in passaged cells. In addition to hedgehog signalling, cilia themselves were also required for a range of pathways, including the mechanical regulation of gene expression and matrix production. Therefore, the loss of cilia that occured with dedifferentiation might also be influencing other pathways and aspects of cell function important for tissue health and homeostasis. The fact that both mechanical loading and hedgehog signalling promoted redifferentiation and were both dependent on primary cilia, means that cell expansion conditions, which result in loss of primary cilia, are likely to preclude the use of these approaches to 
redifferentiate chondrocytes. Therefore, this study highlighted the importance for tissue engineering approaches of maintaining ciliation.

\section{Additional references}

Holmvall K, Camper L, Johansson S, Kimura JH, Lundgren-Akerlund E (1995) Chondrocyte and chondrosarcoma cell integrins with affinity for collagen type ii and their response to mechanical stress. Exp Cell Res 221: 496-503.

McGlashan SR, Cluett EC, Jensen CG, Poole CA (2008) Primary cilia in osteoarthritic chondrocytes: from chondrons to clusters. Dev Dyn 237: 2013-2020.

McGlashan SR, Knight MM, Chowdhury TT, Joshi P, Jensen CG, Kennedy S, Poole CA (2010) Mechanical loading modulates chondrocyte primary cilia incidence and length. Cell Biol Int 34: 441-446.
Rosenzweig DH, Matmati M, Khayat G, Chaudhry S, Hinz B, Quinn TM (2012) Culture of primary bovine chondrocytes on a continuously expanding surface inhibits dedifferentiation. Tissue Eng Part A 18: 24662476.

Thompson CL, Chapple JP, Knight MM (2014) Primary cilia disassembly down-regulates mechanosensitive hedgehog signalling: a feedback mechanism controlling adamts- 5 expression in chondrocytes. Osteoarthritis Cartilage 22: 490-498.

Wong M, Siegrist M, Goodwin K (2003) Cyclic tensile strain and cyclic hydrostatic pressure differentially regulate expression of hypertrophic markers in primary chondrocytes. Bone 33: 685-693.

Editor note: The scientific editor for this paper was Martin Stoddart. 